\title{
A comparison of visible wavelength reflectance hyperspectral imaging and Acid Black 1 for the detection and identification of blood stained fingerprints
}

\begin{abstract}
Bloodstains are often encountered at scenes of violent crime and have significant forensic value for criminal investigations.

Blood is one of the most commonly encountered types of biological evidence and is the most commonly observed fingerprint contaminant. Presumptive tests are used to test blood stain and blood stained fingerprints are targeted with chemical enhancement methods, such as acid stains, including Acid Black 1, Acid Violet 17 or Acid Yellow 7. Although these techniques successfully visualise ridge detail, they are destructive, do not confirm the presence of blood and can have a negative impact on DNA sampling.

A novel application of visible wavelength hyperspectral imaging (HSI) is used for the noncontact, non-destructive detection and identification of blood stained fingerprints on white tiles both before and after wet chemical enhancement using Acid Black 1. The identification was obtained in a non-contact and non-destructive manner, based on the unique visible absorption spectrum of haemoglobin between 400 and $680 \mathrm{~nm}$. Results from the exploration of the selectivity of the setup to detect blood against ten other non-blood protein contaminants are also presented. A direct comparison of the effectiveness of HSI with chemical enhancement using Acid Black 1 on white tiles is also presented.
\end{abstract}

\section{Keywords}

Fingerprints, Blood stained fingerprints, Blood detection, Hyperspectral Imaging, Acid Black 1

\author{
Abbreviations \\ AB- Acid Black 1 \\ DS- Detect stain \\ DSLR- Digital single lens reflex \\ HSI- Hyperspectral imaging \\ PRD- Partial ridge detail \\ WRD- Whole ridge detail
}




\section{Contents}

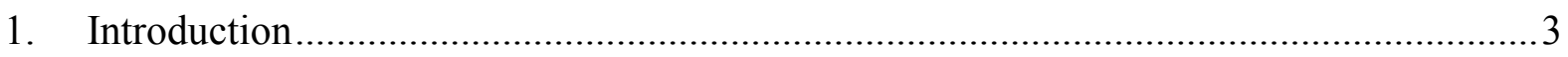

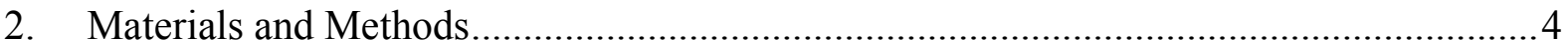

2.1 Contamination of digit and substrate preparation ................................................ 4

2.2 The hyperspectral imaging (HSI) system ....................................................... 4

2.3 Hyperspectral reflectance image acquisition and pre-processing ............................ 4

2.4 Criteria for the identification of blood stains ......................................................... 5

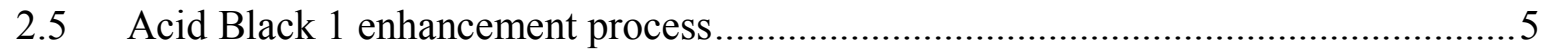

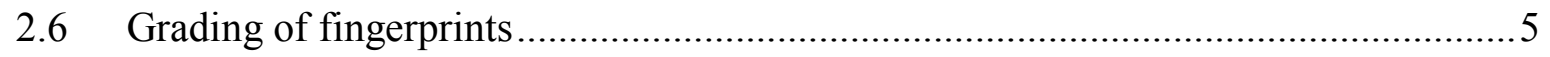

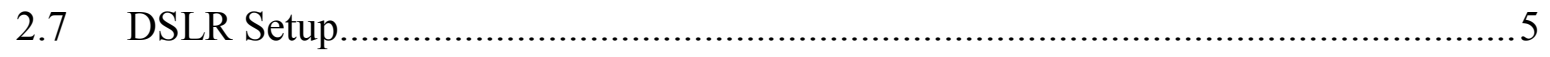

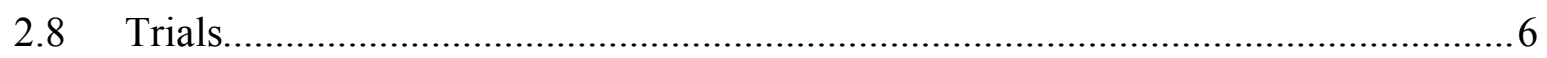

2.8.1 HSI analysis before and after Acid Black enhancement ...................................6

2.8.2 Blood stained fingerprints and other non-blood proteins ................................. 6

2.8.3 HSI analysis compared with Acid Black enhancement .................................... 6

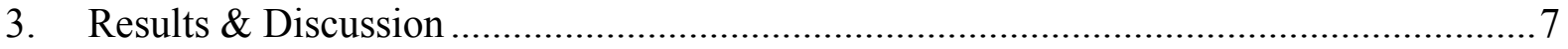

3.1 HSI analysis before and after Acid Black enhancement ......................................... 7

3.2 Blood stained fingerprints and other non-blood proteins .......................................

3.3 HSI analysis compared with Acid Black enhancement ......................................

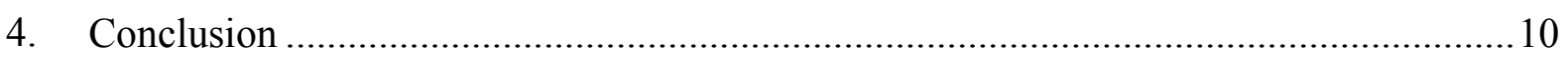

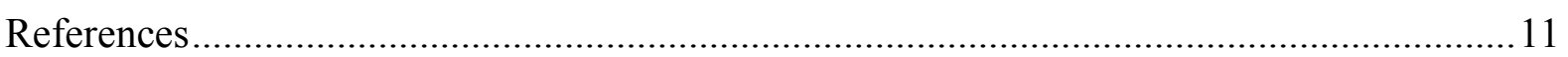




\section{Introduction}

Bloodstains are often encountered at scenes of violent crime and have significant forensic value [1]. Blood is one of the most commonly encountered types of biological evidence [2]. Analysis of the blood spatter pattern can help with reconstructing events and DNA profiling can be carried out, which may assist to establish the identity of the suspect.

The current forensic workflow for the examination of suspected blood stains starts with visual examination, including the use of alternate light sources, to confirm the identity of the substance before further analysis [3]. This is followed by analysis using chemical presumptive tests to provide an indication as to the presence of blood [4]. Presumptive tests are however not specific to blood and can generate false positives [4]. Contamination of the stain may also occur, such as altering the shape of the stain or dilution, which may affect subsequent DNA analysis [5].

The examination of fingerprint evidence also routinely involves the analysis and identification of biological samples, as blood is the most commonly observed fingerprint contaminant [4]. Fingerprints contaminated with blood are subject to chemical enhancement methods depending on the substrate, including acid stains such as Acid Black 1, Acid Violet 17 or Acid Yellow 7 [4]. These chemical enhancement techniques, although successful, have several disadvantages. The most significant is their potentially destructive nature, as incorrect application of the enhancement process will result in a loss of ridge detail [4]. There is also a risk of background staining obscuring any enhanced ridge detail, which can be affected by the substrate nature. Porous surfaces are less suitable for enhancement with Acid black, as considerable background staining can occur [6]. The enhancement methods are also purely presumptive, as successful enhancement of a blood stained fingerprint does not conclusively confirm the presence of blood, due to the potential for false positives from other non-blood proteins. Additionally the staining process may interfere with subsequent recovery of DNA material from the fingerprint.

Considerable previous research has explored the detection and identification of blood stains using a variety of techniques, including RNA analysis [7,8]; microscopy techniques [9], and spectroscopic methods [10-13].

Recent research has explored the use of non-contact reflectance spectroscopy to detect blood stains with high levels of specificity [1]. This led to the use of visible wavelength hyperspectral imaging (HSI), which was first reported for the detection and age determination of horse blood stains between $442 \mathrm{~nm}$ and $585 \mathrm{~nm}$ as proof of concept research [14]. A similar approach was presented by another research group for the identification of blood stains $[15,16]$, detailing a presumptive method for the rapid non-destructive detection of blood stains.

Most recently our research group a new blood stain identification and age determination approach was proposed based on the Soret $\gamma$ band absorption in haemoglobin $[3,17]$. Previous work has also investigated changes to the Soret band in the UV-Visible spectrum of haemoglobin [18], although this was purely focused on changes to the wavelength of the Soret band peak over time for age determination only, and did not allow for identification or confirmation of blood. The approach carried out by our research group indicated a higher sensitivity and specificity for the detection and identification of blood stains over previously proposed methods [3]. Recent research has since successfully demonstrated the application of this technique for the detection, identification, and visualisation of ridge detail in blood stained fingerprints deposited on white ceramic tiles [19] and a range of other substrates commonly encountered at scenes of crime [20].

To date there has been no comparison between HSI and existing chemical enhancement methods, such as Acid Black. One of the key issues involving the optical analysis of fingerprints is to ensure sufficient ridge detail is captured to allow for comparison of crime 
scene marks against fingerprints from a suspect. A comparison of existing enhancement methods against HSI would allow for the sensitivity of the setup to be measured, which is necessary if HSI is to be further developed [20].

In this study a novel application of hyperspectral imaging (HSI) is used for the non-contact, non-destructive detection and identification of blood stained fingerprints on white tiles both before and after wet chemical enhancement using Acid Black 1. Results from the exploration of the selectivity of the setup to detect blood against ten other non-blood protein contaminants are also presented. A direct comparison of the effectiveness of HSI with chemical enhancement using Acid Black 1 on white tiles is also presented. This is the first time a direct comparison between chemical enhancement methods and the detection of blood stained fingerprints using hyperspectral imaging has been explored. Results potentially indicate a significant step towards a reliable method for both non-destructive blood identification and the detection of ridge detail in blood stained fingerprints, both before and after chemical enhancement.

This work is following on from [19,20], where hyperspectral imaging was demonstrated for the first time to detect, identify, and visualise to a sufficient resolution the ridge detail of blood stained fingerprints across a wide range of substrates. The research presented in this paper again demonstrates the potential of HSI, through a comparison of the successful nondestructive detection and identification of blood stained fingerprints against Acid Black chemical enhancement.

\section{Materials and Methods}

\subsection{Contamination of digit and substrate preparation}

Horse blood were used as the contaminant in this study and was deposited into a Petri dish containing a small sponge. The right middle finger was pressed against the sponge to evenly coat the digit and the blood stained fingerprint then deposited onto the white tile. Application of other non-blood protein contaminants was carried out by applying a small quantity to a gloved left middle finger. The contaminant was then evenly spread over the ridge detail on the right middle finger and the fingerprint deposited. All white ceramic tiles (B\&Q, UK) were cleaned with distilled water and thoroughly dried before labelling and fingerprint deposition.

\subsection{The hyperspectral imaging (HSI) system}

The HSI system used in this study was the same setup detailed in [3] and [19,20], consisting of a liquid crystal tuneable filter (LCTF) coupled to a 2.3 megapixel Point Grey camera and a light source for scene illumination. The light source was comprised of two 40W LEDs; one violet giving an output at 410nm and one white giving an output between 450 and $700 \mathrm{~nm}$. Control of the LCTF and image capture was performed using custom developed software written in C++ (Microsoft, USA). Images were captured between $400 \mathrm{~nm}$ and $680 \mathrm{~nm}$ with spectral sub sampling at $5 \mathrm{~nm}$ intervals, resulting in an image cube at 56 wavelengths for each scan. Spectra from the image cube were subsequently analysed using custom routines developed in Visual Studio (Microsoft, USA) and Spyder (Python, USA). The time required to acquire and process an image was approximately 30 seconds.

\subsection{Hyperspectral reflectance image acquisition and pre-processing}

The hyperspectral reflectance measurements were made following the method detailed in [3] and $[19,20]$. A reference image $\left(\mathrm{R}_{0}\right)$ was obtained using a blank ceramic tile. This image was recorded in a $5 \mathrm{~nm}$ series of 56 discrete wavelengths between $400 \mathrm{~nm}$ and $680 \mathrm{~nm}$. The 
sample image $\left(\mathrm{R}_{\mathrm{s}}\right)$ was recorded at the same wavelengths under the same illumination conditions and integration time settings on the camera. The hyperspectral reflectance image (R) consisted of a data cube of $1280 \times 1024$ pixel values at 56 discrete wavelengths. Additional information regarding sample image processing can be found in [3].

\subsection{Criteria for the identification of blood stains}

Haemoglobin dominates the blood reflectance spectrum in the visible region $[14,17]$. The spectrum contains a strong narrow absorption at $415 \mathrm{~nm}$ called the Soret or $\gamma$ band with two weaker and broader absorptions between 500 and $600 \mathrm{~nm}$ known as the $\beta$ and $\alpha$ bands [3]. Due to absorption in the blue part of the visible spectrum, the Soret band results in the distinctive red colour of blood. Other red substances also absorb between 400 and $680 \mathrm{~nm}$ in the blue region, although these absorption features tend to not be centred at $415 \mathrm{~nm}$ and are much broader. This is the basis of the methodology used in this study to identify and discriminate blood from other similarly coloured substances. Further information is detailed in [3]. From the reflectance images obtained, the pixels which satisfied the criterion were marked as black, whilst all other pixels were marked as white. This allowed regions of the image where the blood stained fingerprint was present to be identified, as well as clear distinction of the ridge detail.

\subsection{Acid Black 1 enhancement process}

Deposited blood stained fingerprints were enhanced using a 3 stage water-based Acid Black 1 (Amido black 10B) process, as proposed by CAST [4]. The ceramic tiles were submerged in a fixing solution composed of 5-sulphosalicylic acid $2 \mathrm{~g} / 100 \mathrm{~mL}(\%)$ and distilled water for 5 minutes and then submerged in a staining solution composed of acid dye $0.2 \mathrm{~g} / 100 \mathrm{~mL}(\%)$, acetic acid $5 \% \mathrm{v} / \mathrm{v}$ and distilled water for 4 minutes. After staining, the ceramic tiles were rinsed using a washing solution composed of acetic acid 5\% v/v and distilled water. The enhanced fingerprints were then left to dry at room temperature for at least 30 minutes before photography or further analysis.

\subsection{Grading of fingerprints}

Blood stained fingerprints were assessed on three criteria:

i. The furthest depletion where it was possible to detect ridge detail across the whole of fingerprint (whole ridge detail, WRD)

ii. The furthest depletion where it was possible to detect some ridge detail (partial ridge detail, PRD)

iii. The furthest depletion where it was possible to detect the blood stain but no ridge detail could be resolved (detect stain, DS).

This grading method was used by the same experienced investigator for all fingerprints and was used in preference to the normal ordinal scale [21], as the focus of the study was on the sensitivity of HSI as an analysis technique compared to DSLR and Acid Black enhancement. The further down the depletion series ridge detail could be detected across the whole fingerprint, the higher the sensitivity of the technique.

\subsection{DSLR Setup}

The images used in this report were taken using a digital single lens reflex (DSLR) camera mounted on a Kaiser RS1 copy stand. The DSLR was a Canon EOS 700D which was fitted with a Canon Angle Finder C $90^{\circ}$ viewfinder with a $1.25-2.5 x$ optical magnification and a Canon TC-80N3 remote control external shutter release to avoid camera motion. Images were taken using two sizes of macro lenses- a $50 \mathrm{~mm}$ lens for overview shots of the substrates and a 
$100 \mathrm{~mm}$ lens for high magnification macro shots of individual fingerprints. The $50 \mathrm{~mm}$ lens was used as it is recognised as being generally equivalent to the view seen by the human eye [22]. The lenses used were a Canon EF $50 \mathrm{~mm}$ f 2.5 macro lens and a Canon EF $100 \mathrm{~mm}$ f $2.8 \mathrm{~L}$ macro IS USM lens. Substrates were lit using oblique lighting from two Daylight Twist Portable Lamps with a white light output of $6500 \mathrm{~K}$.

\subsection{Trials}

Three trials were designed to explore fingerprint detection and realisation of ridge detail using HSI in comparison to ridge detail visible by DSLR and after Acid Black 1 chemical enhancement. All fingerprints were deposited by one male donor (age 26, omnivore, no cosmetics).

\subsubsection{HSI analysis before and after Acid Black enhancement}

Four white ceramic tiles were prepared and depletion series of 12 fingerprints in undiluted horse blood were deposited onto the tiles. Each tile was photographed and then analysed using HSI. The tiles were enhanced using the 3 stage water-based Acid Black 1 process detailed in $\mathbf{2 . 5}$, as proposed by CAST [4]. The ceramic tiles were submerged in the fixing solution for 5 minutes and the staining solution for 4 minutes. The ceramic tiles were rinsed using the wash solution and then allowed to dry for at least 30 minutes. The tiles were then re-photographed and analysed using HSI for comparison against DSLR and Acid Black. The sequential process shown in Figure 1 details the double HSI analysis carried out, both before and after enhancement.

[Insert Figure 1]

Figure 1: Double analysis flowchart of DSLR, HSI and Acid Black 1 enhancement

\subsubsection{Blood stained fingerprints and other non-blood proteins}

Acid Black 1 is a protein stain and will enhance both blood and other protein contaminants. The HSI setup is specific to blood and will only visualise stains or fingerprint ridge detail in blood. To date there have been zero false positives across all substrates explored [3]. To establish the accuracy of the HSI setup at identifying blood fingerprints, two fingerprints were deposited using undiluted horse blood onto a white ceramic tile. An additional 10 fingerprints contaminated with 7 different protein contaminants, were deposited onto the same tile, as detailed in Table 1. Analysis was carried out following the same procedure as detailed in 2.8.1, and analysis was carried out both before and after Acid Black enhancement. HSI analysis was therefore again carried out in a double analysis sequential process, as shown in Figure 1, so as to identify the effect of non-blood proteins on both HSI analysis and Acid Black enhancement.

[Insert Table 1]

Table 1: Protein contaminants and protein content (protein contaminants from packaging, horse blood from [23])

\subsubsection{HSI analysis compared with Acid Black enhancement}

Depletion series of twelve fingerprints in undiluted human blood were deposited onto ten white ceramic tiles. Analysis was carried out following the same procedure as detailed in 2.8.1 with analysis was carried out before Acid Black enhancement, as shown in Figure 2. This allowed for a comparison of the sensitivity of HSI analysis with chemical enhancement using Acid Black. 
[Insert Figure 2]

Figure 2: Flowchart of DSLR, HSI and Acid Black 1 enhancement

\section{Results \& Discussion}

\subsection{HSI analysis before and after Acid Black enhancement}

The ability of HSI to identify blood stained fingerprints both before and after chemical enhancement using Acid Black 1 was explored and demonstrated considerable advantages to the HSI setup. Analysis of the twelve depletions using HSI detected ridge detail to the eighth depletion and the stain to the twelfth depletion across all 4 tiles, as shown in Figure 3. After enhancement with Acid Black 1, HSI was still able to detect ridge detail to the eighth depletion, as shown in Figure 4. Therefore analysis after chemical enhancement with Acid Black did not affect the ability to detect ridge detail in blood stained fingerprints.

The limit of detection was reduced after enhancement, as the stain was could only be detected to the tenth depletion with some noise, indicating enhancement results in a slight reduction in HSI sensitivity. This is not unexpected however, as the additional material present over the blood stained fingerprint due to enhancement was anticipated to mask the absorption spectrum of blood. This masking effect appeared to have the largest impact for lower level depletions, which is most likely due to the reduced concentration of blood present.

\section{[Insert Figure 3]}

Figure 3: Images of 12 print depletions on white tile prior to Acid Black 1 enhancement: DSLR (left) and HSI images overlaid

[Insert Figure 4]

Figure 4: Images of 12 print depletions on white tile after Acid Black 1 enhancement: DSLR (left) and HSI images overlaid (right)

\subsection{Blood stained fingerprints and other non-blood proteins}

Acid Black 1 is a protein stain and will enhance both blood and other non-blood protein contaminants, such as egg white [4]. The HSI setup is specific to blood and will only visualise stains or blood stained fingerprint ridge detail. This specificity was thoroughly tested in this trial, with blood stained fingerprints deposited alongside fingerprints in other non-blood protein contaminants. Using visual examination alone, the blood stained fingerprints were difficult to distinguish from the other protein contaminants, due to the similarity of the red/brown colour. However the blood fingerprints are easily identifiable in the hyperspectral images, as shown in Figure 5, as only the blood stained fingerprints deposited in positions six and ten are detected. The ten other non-blood contaminants were not detected using HSI resulting in a blank white image after HSI analysis. This is due to the significantly different absorption spectra of blood compared to the protein contaminants, as shown in Figure 6. A magnified overlay shows the spectra of the non-blood contaminants. Wey powder and egg white have a small broad peak at $415 \mathrm{~nm}$ but only the spectrum of blood has the sharp Soret band, which forms the basis for the identification method demonstrated in this paper. Out of all the protein contaminants, the spectrum of marmite has the most significant increase around $415 \mathrm{~nm}$, although the absorbance increase around $430 \mathrm{~nm}$ is considerably less sharp than the Soret band observable in the blood stain. 
After Acid Black enhancement, all 12 deposited fingerprints were stained blue, indicating the possible presence of blood. In the hyperspectral image, the blood fingerprints are still clearly identifiable as depositions six and ten, despite chemical treatment over the top, as shown in Figure 7. Again, only the blood stained fingerprints were detected using HSI and the ten other non-blood contaminants yielded a blank white image. In the DSLR image after enhancement, deposition four is no longer visible, and eight and eleven are very faint, as a significant quantity of the marmite and fish paste contaminants were washed off the substrate during the Acid Black enhancement process. As a result, significant ridge detail was potentially lost, which highlights the disadvantages of chemical enhancement methods and reinforces the need for a non-destructive method for fingerprint analysis.

This is demonstrated as a significant benefit of the HSI system- it can non-destructively analyse a suspected blood stained fingerprint to confirm the presence of blood and does not produce false positives due to the presence of other contaminants. Acid Black however has several drawbacks, as it involves direct contact with a stain, is destructive, and involves a three-step chemical treatment, which is not a quick process. Additionally, Acid Black can react with other compounds, so a positively enhanced fingerprint does not necessarily indicate that blood was present.

[Insert Figure 5]

Figure 5: Images of 12 contaminants on white tile prior to Acid Black 1 enhancement: DSLR (left) and HSI images overlaid (right)

[Insert Figure 6]

Figure 6: Absorption spectra of blood and protein contaminants between 400 and $680 \mathrm{~nm}$

[Insert Figure 7]

Figure 7: Images of 12 contaminants on white tile after Acid Black 1 enhancement: DSLR (left) and HSI images overlaid (right)

\subsection{HSI analysis compared with Acid Black enhancement}

The sensitivity of HSI compared to DSLR photography and Acid Black 1 was explored using the level of ridge detail visible in fingerprint depletions on white tiles.

DSLR had the lowest mean score across all depositions, with no ridge detail visible after the 6th depletion and the stain not observable after the 9th depletion, as shown in Figure 8 . HSI scored similar to DSLR for the first 5 depletions, after which it was significantly better $(\mathrm{p}<$ 0.001) at both detection of ridge detail and the stain, as shown in Table 2. HSI was also significantly better $(p=0.004)$ than DSLR for the 3rd depletion as ridge detail was still detected across the whole of the fingerprint. Acid Black had the highest mean score across all depositions, as shown in Figure 8, and was statistically better than DSLR from the 3rd depletion onwards $(\mathrm{p}=0.004)$. Acid Black was also statistically better than HSI from the 4th depletion onwards $(p<0.001)$, although not for the 9th depletion $(p=0.051)$. Analysis using Kruskal-Wallis identified each method as statistically different $(p<0.001)$, as shown in Figure 9.

Overall HSI is comparable to DSLR for the detection of whole ridge detail and significantly better for detection of partial ridge detail and to detect the stain. Acid Black is the best method overall for both visualisation of ridge detail and detection of the stain. A comparison of one set of the images produced is shown in Figure 10. However, the difference between HSI and Acid Black was smaller for detection of partial ridge detail and detection of the stain compared to detection of whole ridge detail. 
Overall the comparison shows that HSI can visualise ridge detail better than DSLR, but not as well as chemical enhancement using Acid Black. This high level of sensitivity of Acid Black is expected as the Acid Black method is commonly reported as being highly sensitive and is recommended as a key enhancement reagent for visualising blood fingerprints [4]. However improvements have already been made to the HSI setup, including a camera with a higher resolution than the 2.3 megapixel camera used currently to improve both the sensitivity and the amount of ridge detail observable. This would therefore improve the depletion to which both full and partial ridge detail could be detected, resulting in a more comparable technique to chemical enhancement using Acid Black. The difference in sensitivity between HSI and Acid Black for the detection of blood stains is smaller than the difference for the visualisation of ridge detail. Increased sensitivity to the detection of ridge detail has already been demonstrated through improvements to the HSI setup [20], including increasing the camera resolution from the current 2.3 megapixels.

The use of HSI has other advantages over chemical enhancement however, as it is not only non-contact and non-destructive, but a single scan is considerably faster than a three-step chemical treatment like Acid Black. HSI can also confirm that a red contaminant is blood, whereas Acid Black can react with other compounds such as egg white [4] to generate false positives, so an enhanced fingerprint does not necessarily indicate blood was present.

[Insert Figure 8]

Figure 8: A comparison of the mean scores for DSLR, HSI and Acid Black for each of the 12 depletions

[Insert Table 2]

Table 2: Comparing DSLR, HSI and Acid Black enhancement using the Mann-Whitney $U$ test for each depletion ( $p<0.05$ highlighted green)

[Insert Figure 9]

Figure 9: Comparing the overall score from DSLR (Test 1), HSI (Test 2) and Acid Black enhancement (Test 3) using Kruskal-Wallis $(p<0.05)$

[Insert Figure 10]

Figure 10: A comparison of the blood DSLR image (left), Hyperspectral image (centre) and the Acid Black enhanced DSLR image (right) for 12 depletions 


\section{Conclusion}

A comparison of DLSR and chemical enhancement using Acid Black with visible wavelength reflectance hyperspectral imaging has been carried out for the non-contact and nondestructive detection and positive identification of blood stained fingerprints on white tiles. In the processed hyperspectral images, pixels where blood was identified were coloured black whilst all other pixels were coloured white, thus enhancing the location of ridge detail in blood stained fingerprints.

This is the first time that visible wavelength hyperspectral imaging has been directly compared with existing chemical enhancement methods for the visualisation of blood stained fingerprints. The results demonstrate potential considerable advantages of HSI over existing chemical enhancement methods, as hyperspectral analysis is both non-contact and nondestructive, thereby minimising the potential for damage to the ridge detail currently possible with wet chemical presumptive tests. Research also identified that hyperspectral analysis is not adversely affected by chemical enhancement and confirmation of blood using HSI can be carried out both prior and subsequent to chemical enhancement. This demonstrates a significant benefit to the HSI method, as non-destructive hyperspectral analysis could be used to confirm an enhanced fingerprint is actually blood. HSI also demonstrated an extremely high selectivity to blood, as analysis successfully identified two blood stained fingerprints deposited alongside ten fingerprints in other non-blood protein contaminants. This high level of selectivity was achieved both before and after Acid Black 1 enhancement, despite Acid Black producing a large number of false positives and destroying the ridge detail of some deposited fingerprints. A comparison of DSLR, HSI and chemical enhancement using Acid Black determined that HSI can visualise ridge detail better than DSLR photography, but not as well as chemical enhancement using Acid Black. However improvements have since been made to the HSI setup, including the installation of a higher resolution camera than the current 2.3 megapixel camera, so as to improve both the sensitivity and the amount of ridge detail observable. This would therefore improve the depletion to which both full and partial ridge detail could be detected, resulting in a more comparable technique to chemical enhancement using Acid Black. Further research is therefore required to compare the improved HSI Version 2 setup against current chemical enhancement methods, including alternate formulations.

Overall HSI has significant benefits for the detection and identification of blood stained fingerprints and blood stains. Further work is required to compare the technique against other existing chemical enhancement methods across a wide range of substrates, so as to explore the potential of implementing HSI within the existing forensic workflow for blood detection and identification. Additionally the application of previous research exploring the age determination of blood stains could be applied to blood stained fingerprints to determine the age of the blood with which the fingerprint was deposited. Development of a more rugged instrument could lead on to a robust portable device which could be used at crime scenes. This would then allow for both the non-contact, non-destructive identification and visualisation of ridge detail across a wide range of substrates, both before and after chemical enhancement, as well as the potential to determine the age of blood in blood stained fingerprints. 


\section{References}

[1] Rolf H. Bremmer, Annemarie Nadort, Ton G. van Leeuwen, Martin J. C. van Gemert, Maurice C. G. Aalders, Age estimation of blood stains by hemoglobin derivative determination using reflectance spectroscopy, Forensic science international, 206, 1-3, (2011), 166-171

[2] Jonathan Finnis, Jennie Lewis, Andrew Davidson, Comparison of methods for visualizing blood on dark surfaces, Science \& Justice, 53, 2, (2013), 178-186

[3] Bo Li, Peter Beveridge, William T. O'Hare, Meez Islam, The application of visible wavelength reflectance hyperspectral imaging for the detection and identification of blood stains, Science \& Justice, 54, 6, (2014), 432-438

[4] Home Office CAST, Fingerprint Sourcebook, Chapter 3, 3.1 Acid Dyes, 1st ed., Home Office, 2013

[5] Neha Passi, Rakesh Kumar Garg, Mukesh Yadav, Ram Sarup Singh, Magdy A. Kharoshah, Effect of luminol and bleaching agent on the serological and DNA analysis from bloodstain, Egyptian Journal of Forensic Sciences, 2, 2, (2012), 54-61

[6] Lydia C. A. M. Bossers, Claude Roux, Michael Bell, Andrew M. McDonagh, Methods for the enhancement of fingermarks in blood, Forensic science international, 210, 1-3, (2011), $1-11$

[7] Kelly Virkler, Igor K. Lednev, Analysis of body fluids for forensic purposes: From laboratory testing to non-destructive rapid confirmatory identification at a crime scene, Forensic science international, 188, 1-3, (2009), 1-17

[8] M. Bauer, RNA in forensic science, Forensic Science International: Genetics, 1, 1, (2007), 69-74

[9] K. De Wael, L. Lepot, F. Gason, B. Gilbert, In search of blood-Detection of minute particles using spectroscopic methods, Forensic science international, 180, 1, (2008), 37-42

[10] Gregory McLaughlin, Vitali Sikirzhytski, Igor K. Lednev, Circumventing substrate interference in the Raman spectroscopic identification of blood stains, Forensic science international, 231, 1-3, (2013), 157-166

[11] Gerda Edelman, Vicky Manti, Saskia M. van Ruth, Ton van Leeuwen, Maurice Aalders, Identification and age estimation of blood stains on colored backgrounds by near infrared spectroscopy, Forensic science international, 220, 1-3, (2012), 239-244

[12] Jacob I. Trombka, Jeffrey Schweitzer, Carl Selavka, Mark Dale, Norman Gahn, Samuel Floyd, James Marie, Maritza Hobson, Jerry Zeosky, Ken Martin, Timothy McClannahan, Pamela Solomon, Elyse Gottschang, Crime scene investigations using portable, nondestructive space exploration technology, Forensic science international, 129, 1, (2002), 1-9

[13] Apollo Chun-Yen Lin, Hsing-Mei Hsieh, Li-Chin Tsai, Adrian Linacre, James Chun-I Lee, Forensic Applications of Infrared Imaging for the Detection and Recording of Latent Evidence, Journal of forensic sciences, 52, 5, (2007), 1148-1150

[14] Bo Li, Peter Beveridge, William T. O'Hare, Meez Islam, The estimation of the age of a blood stain using reflectance spectroscopy with a microspectrophotometer, spectral preprocessing and linear discriminant analysis, Forensic science international, 212, 1-3, (2011), 198-204

[15] Suwatwong Janchaysang, Sarun Sumriddetchkajorn, Prathan Buranasiri, Tunable filterbased multispectral imaging for detection of blood stains on construction material substrates Part 2: realization of rapid blood stain detection, Applied Optics, 52, 20, (2013), 4898-4910

[16] Suwatwong Janchaysang, Sarun Sumriddetchkajorn, Prathan Buranasiri, Tunable filterbased multispectral imaging for detection of blood stains on construction material substrates Part 1: Developing blood stain discrimination criteria, Applied Optics, 51, 29, (2012), 69846996 
[17] Bo Li, Peter Beveridge, William T. O'Hare, Meez Islam, The age estimation of blood stains up to 30 days old using visible wavelength hyperspectral image analysis and linear discriminant analysis, Science \& Justice, 53, 3, (2013), 270-277

[18] Jack Ballantyne, Determination of the Age (Time Since Deposition) of a Biological Stain, Document No 226811, National Institute of Justice Award 2005-MU-BX-K071, (2009) [19] Samuel Cadd, Bo Li, Peter Beveridge, William T. O'Hare, Andrew Campbell, Meez Islam, Non-contact detection and identification of blood stained fingerprints using visible wavelength reflectance hyperspectral imaging: part 1, Science and Justice, (2016),

[20] Samuel Cadd, Bo Li, Peter Beveridge, William T. O'Hare, Andrew Campbell, Meez Islam, The non-contact detection and identification of blood stained fingerprints using visible wavelength hyperspectral imaging: part II effectiveness on a range of substrates, Science and Justice, (2016),

[21] S. Cadd, S. Bleay, V. Sears, Evaluation of the solvent black 3 fingermark enhancement reagent: Part 2 - Investigation of the optimum formulation and application parameters, Science and Justice, 53, 2, (2013), 131-143

[22] Michael Langford, Anna Fox, Richard Sawdon Smith, Langford's Basic Photography, 8th ed., Focal Press, Oxford, 2009

[23] Barbara Riond, Bettina Wenger-Riggenbach, Regina Hofmann-Lehmann, Hans Lutz, Serum protein concentrations from clinically healthy horses determined by agarose gel electrophoresis, Veterinary Clinical Pathology, 38, 1, (2009), 73-77 
Table 1

\begin{tabular}{c|l|l|c} 
Number of prints & Contaminant & \multicolumn{1}{|c|}{ Type/Brand } & Protein content (g) in 100g \\
\hline 2 & Blood & Horse & $29.6-38.5$ \\
2 & Whey powder & 397g, Precision Engineered- Advanced pure whey protein powder- Natural & 75.4 \\
2 & Egg white & Aldi British free-range egg- Medium & 11.0 \\
2 & Marmite & $125 \mathrm{~g}$, Unilever & 39.0 \\
1 & Yogurt & 200g, 0\% fat free Greek style natural yogurt & 7.3 \\
1 & Cream cheese & $250 \mathrm{~g}$, Tesco soft cheese medium fat & 11.0 \\
1 & Meat paste & $75 \mathrm{~g}$, Princes chicken and ham paste & 14.5 \\
1 & Fish paste & $75 \mathrm{~g}$, Princes salmon paste & 12.4
\end{tabular}


Table 2

\begin{tabular}{c|r|r|r} 
Depletion & DSLR vs HSI & HSI vs AB & DSLR vs AB \\
\hline $\mathbf{1}$ & 1.000 & 1.000 & 1.000 \\
$\mathbf{2}$ & 0.317 & 1.000 & 0.317 \\
$\mathbf{3}$ & 0.004 & 1.000 & 0.004 \\
$\mathbf{4}$ & 0.957 & $<0.001$ & $<0.001$ \\
$\mathbf{5}$ & 0.134 & 0.001 & $<0.001$ \\
$\mathbf{6}$ & $<0.001$ & $<0.001$ & $<0.001$ \\
$\mathbf{7}$ & $<0.001$ & 0.001 & $<0.001$ \\
$\mathbf{8}$ & $<0.001$ & 0.028 & $<0.001$ \\
$\mathbf{9}$ & $<0.001$ & 0.051 & $<0.001$ \\
$\mathbf{1 0}$ & $<0.001$ & 0.007 & $<0.001$ \\
$\mathbf{1 1}$ & 0.029 & 0.001 & $<0.001$ \\
$\mathbf{1 2}$ & 0.029 & 0.001 & $<0.001$ \\
\hline Overall & $<0.001$ & $<0.001$ & $<0.001$
\end{tabular}


Figure 1

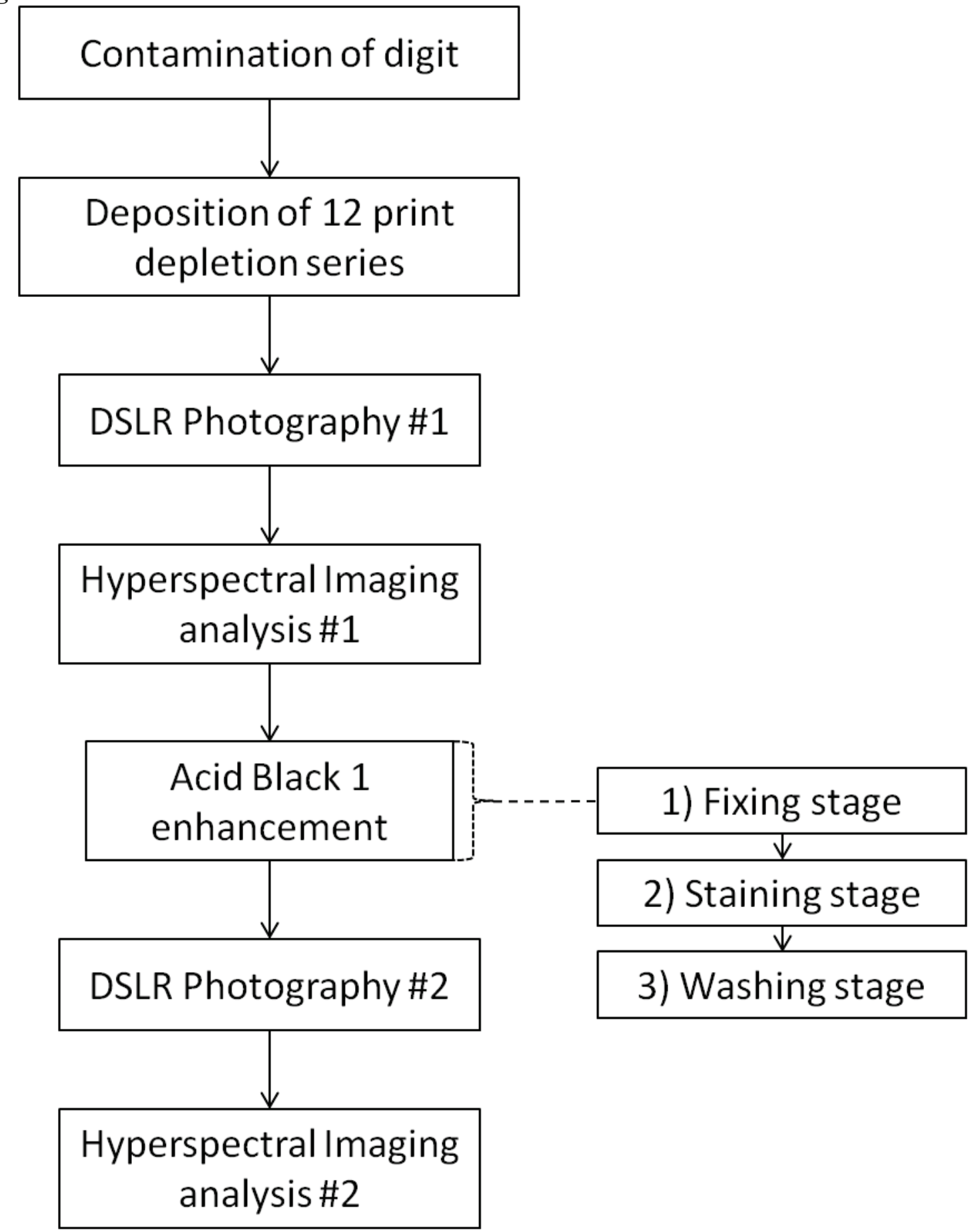


Figure 2

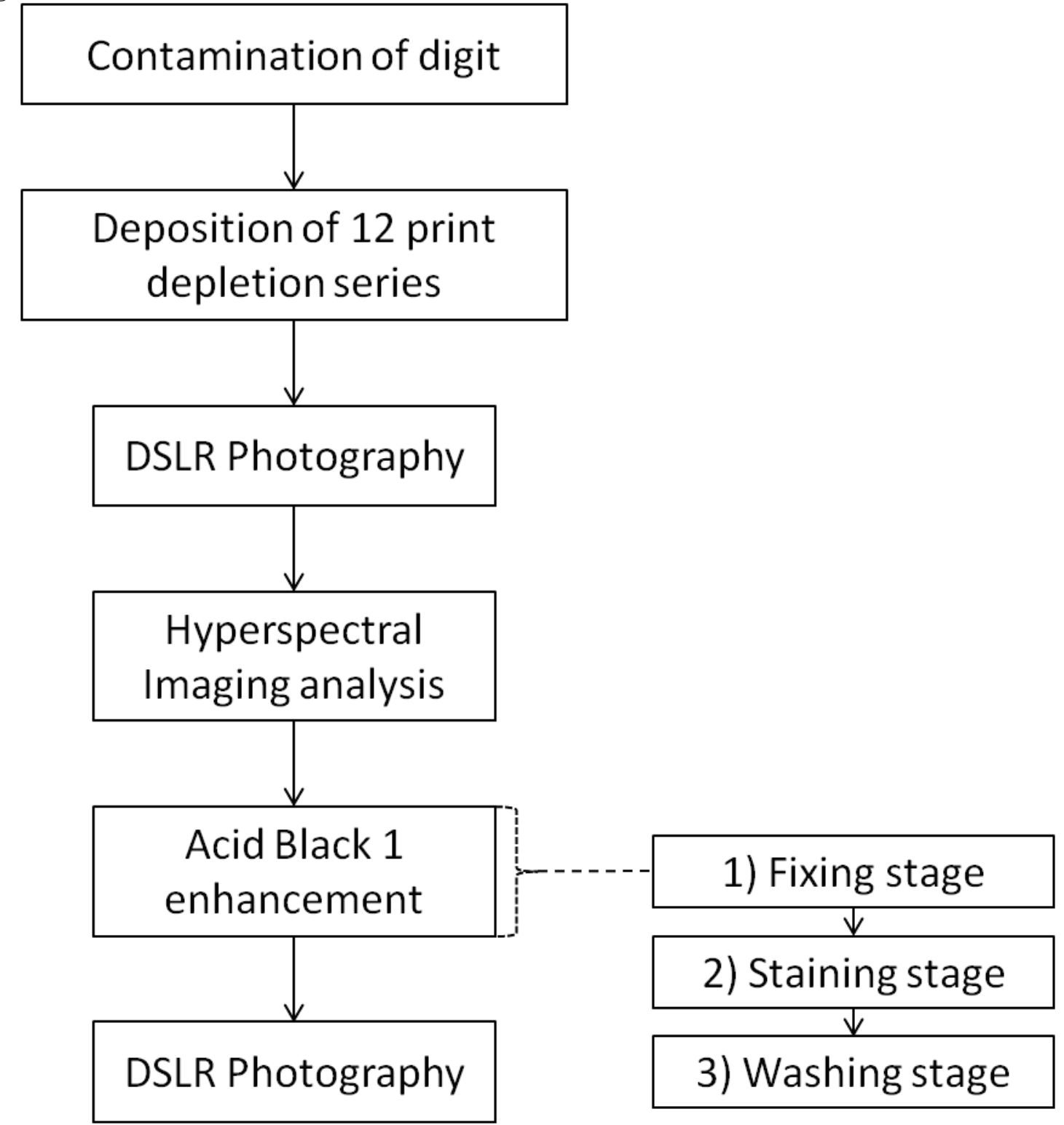


Figure 3

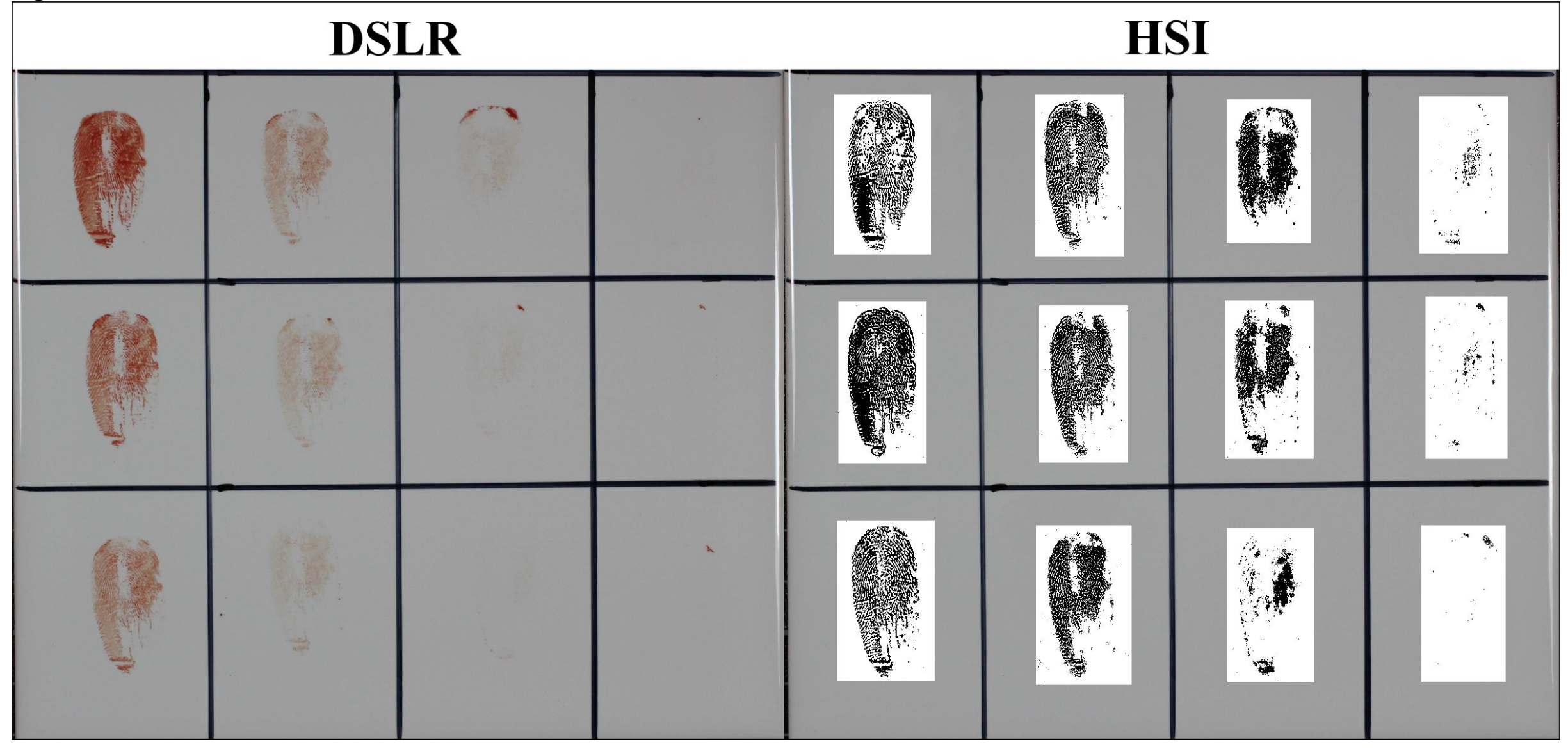


Figure 4

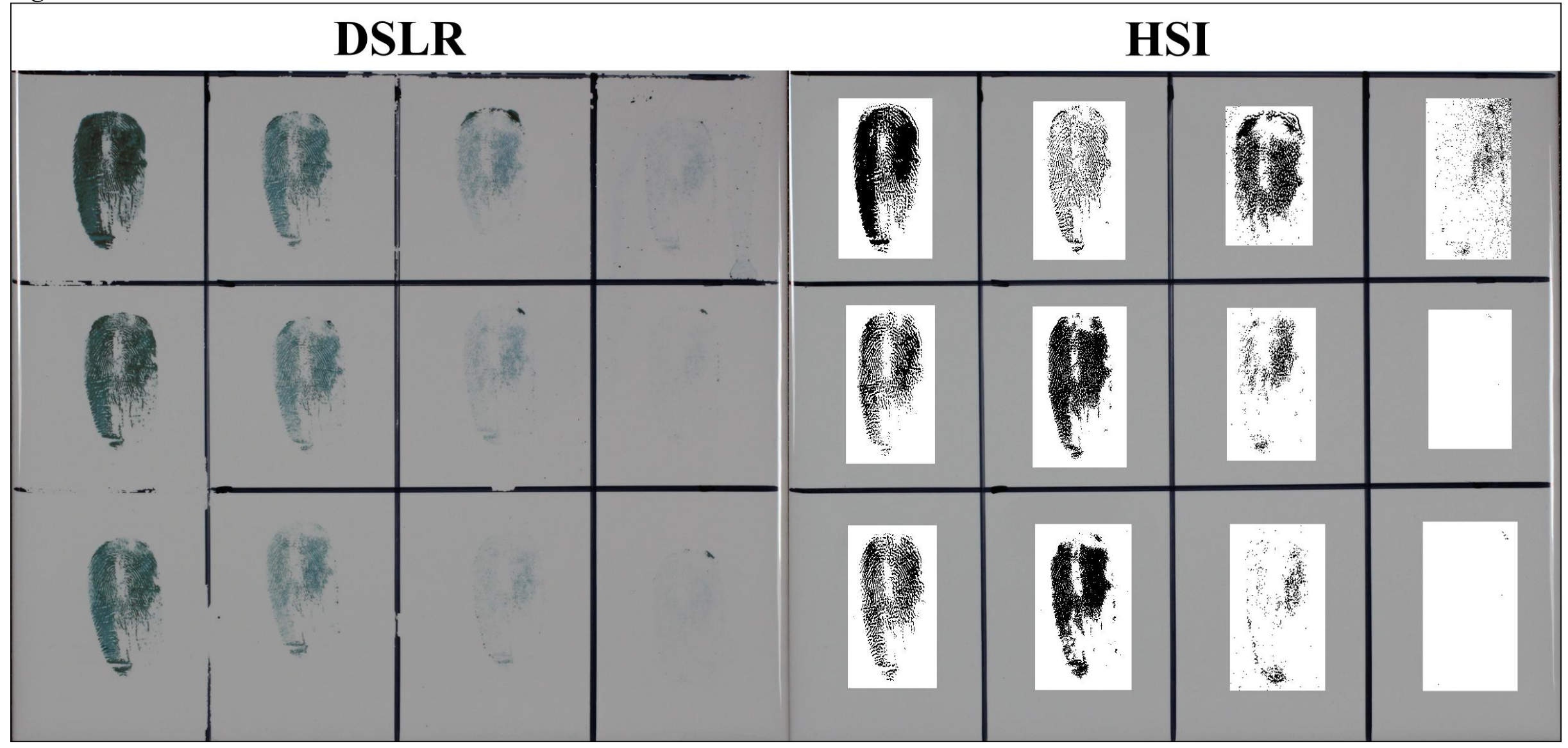


Figure 5

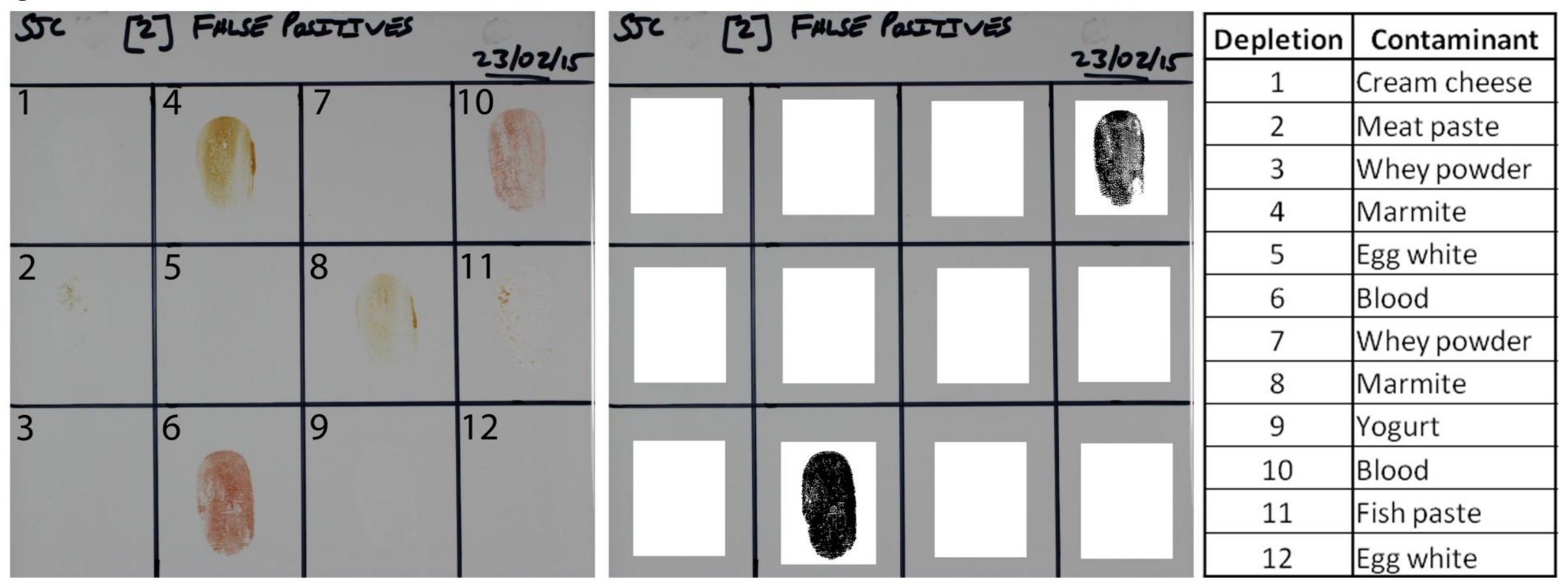


Figure 6

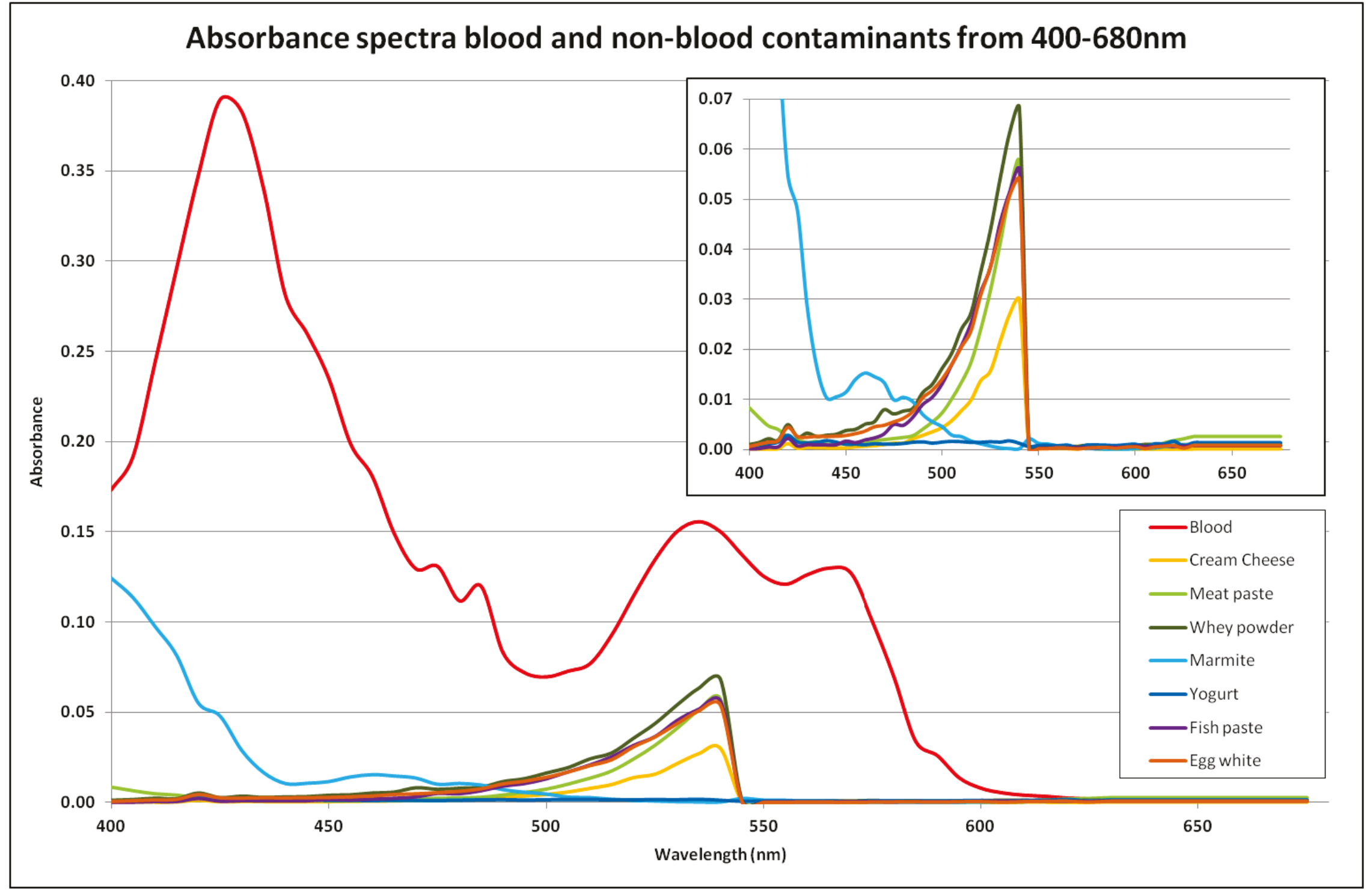

Page 20 of 24 
Figure 7
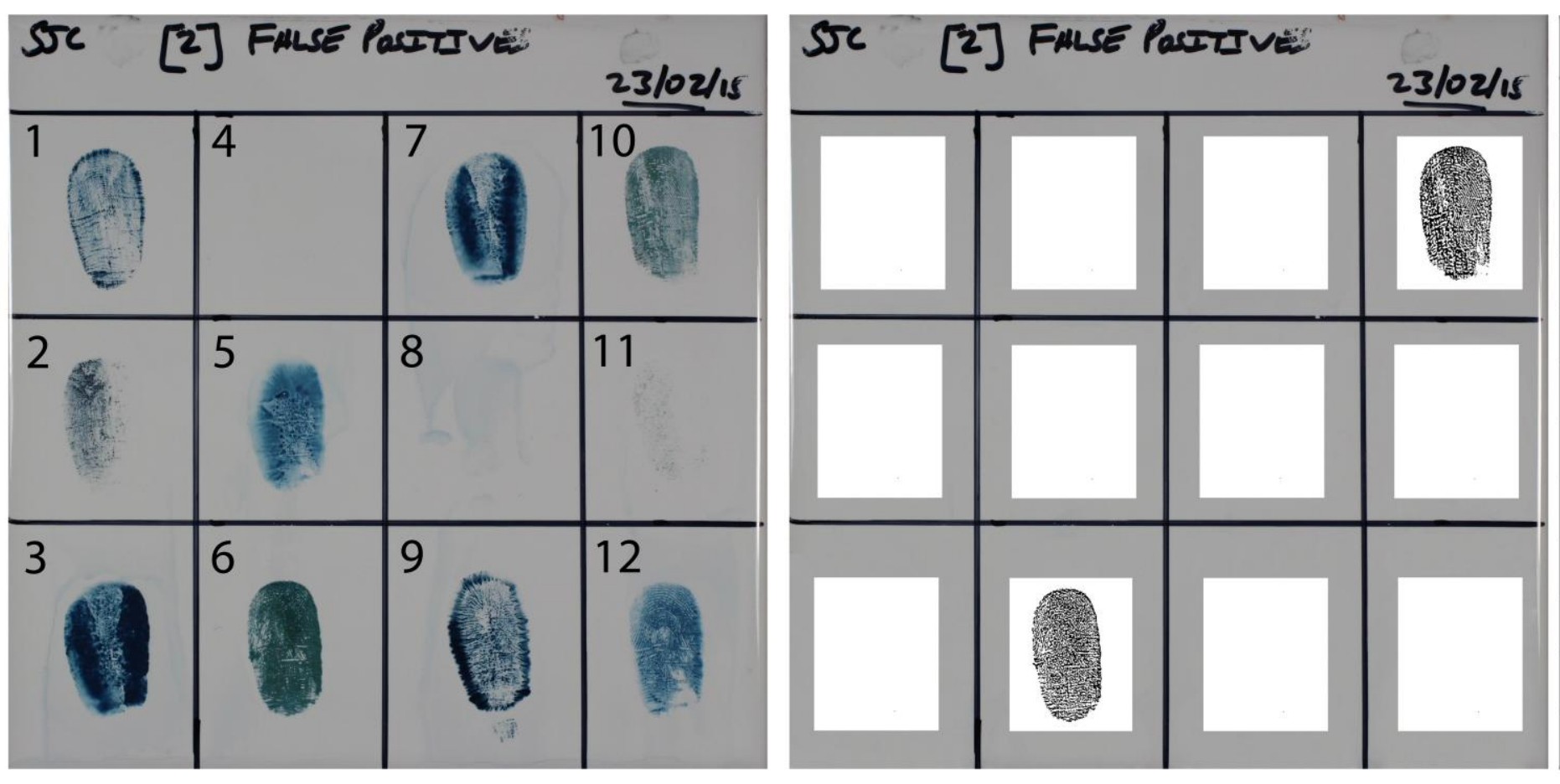

\begin{tabular}{|c|l|}
\hline Depletion & Contaminant \\
\hline 1 & Cream cheese \\
\hline 2 & Meat paste \\
\hline 3 & Whey powder \\
\hline 4 & Marmite \\
\hline 5 & Egg white \\
\hline 6 & Blood \\
\hline 7 & Whey powder \\
\hline 8 & Marmite \\
\hline 9 & Yogurt \\
\hline 10 & Blood \\
\hline 11 & Fish paste \\
\hline 12 & Egg white \\
\hline
\end{tabular}


Figure 8

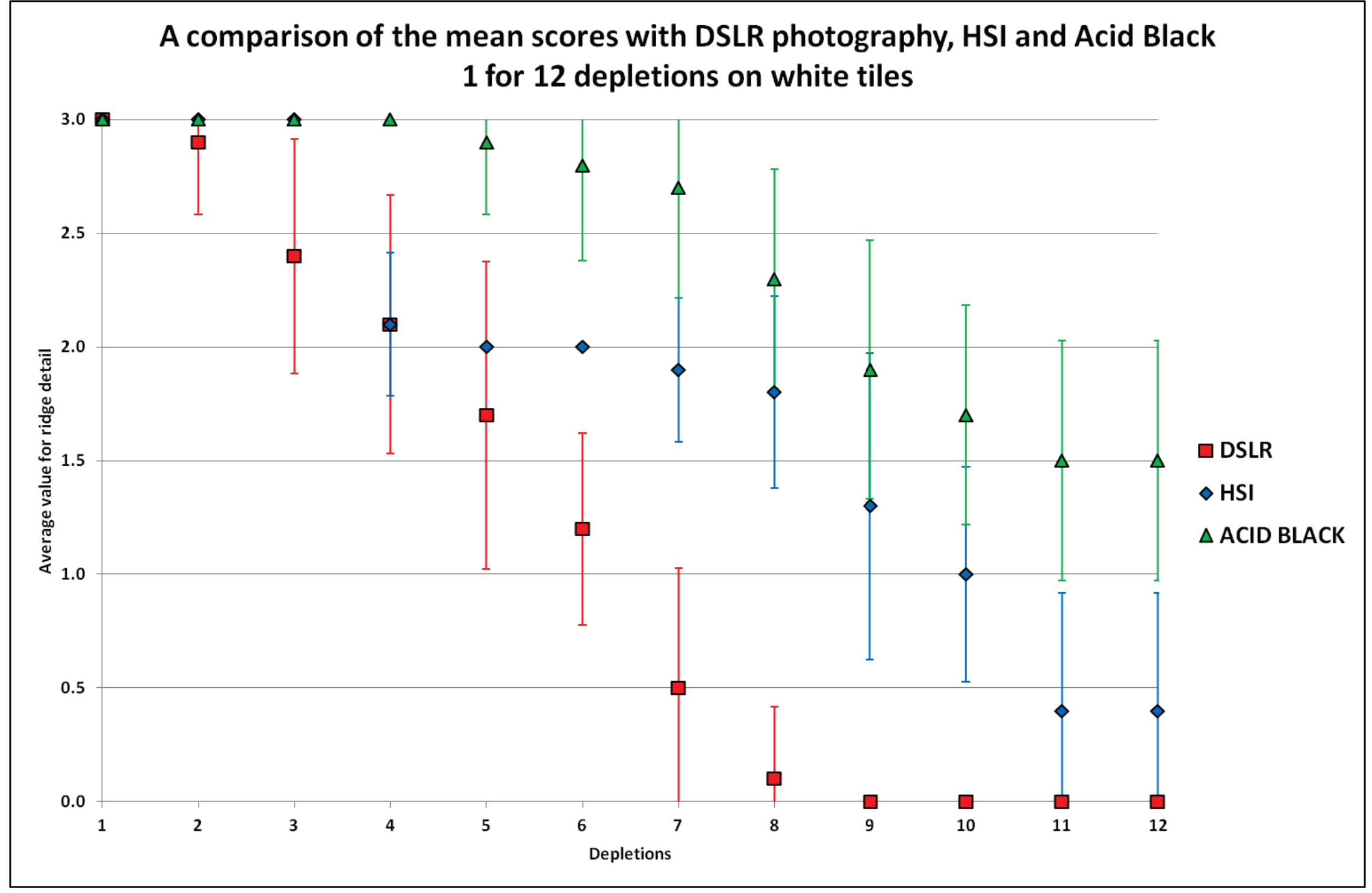

Page 22 of 24 
Figure 9

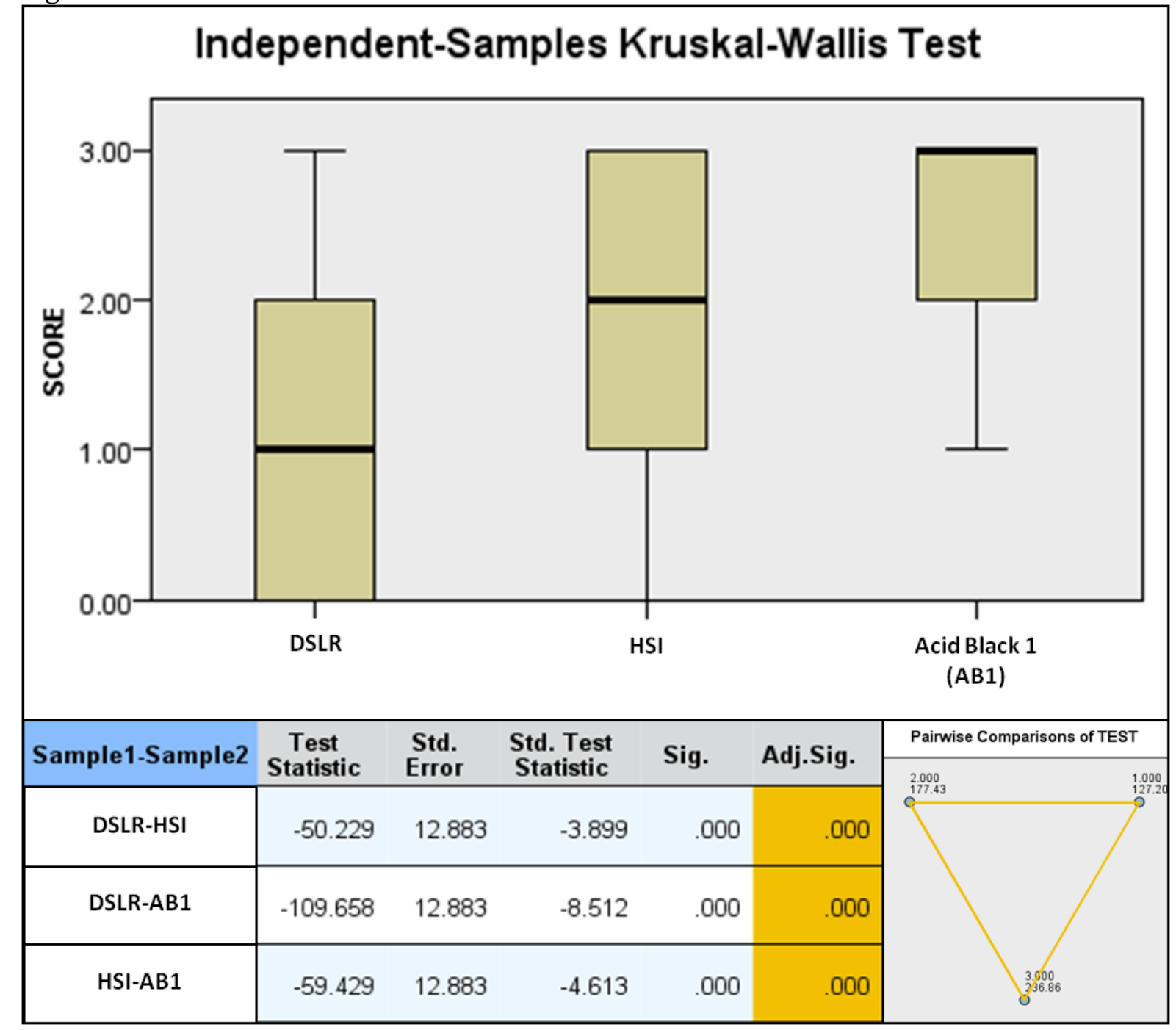


Figure 10

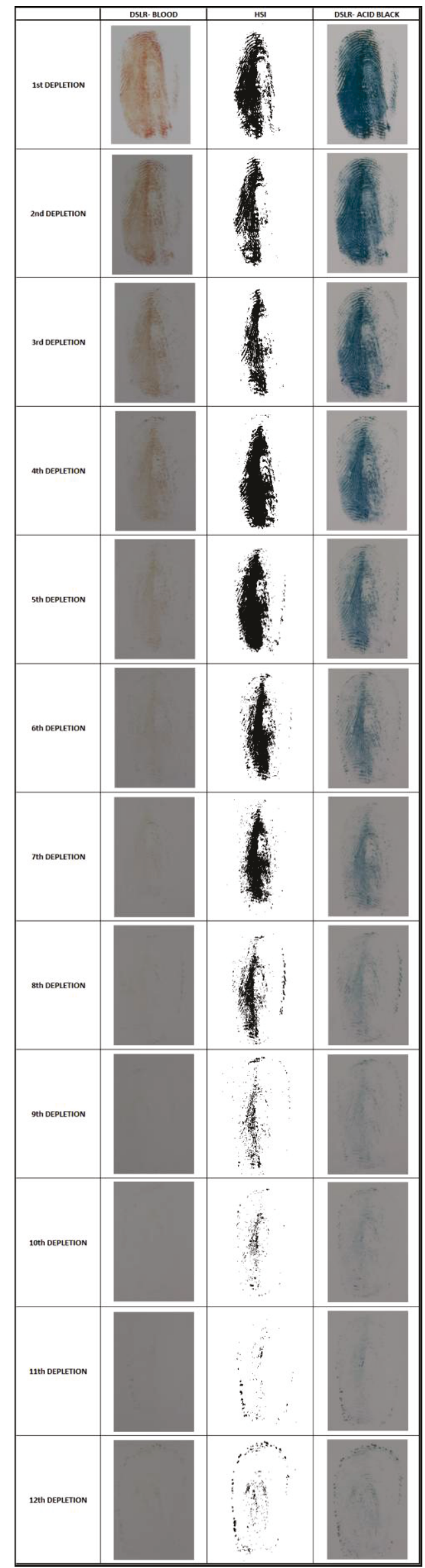

Page 24 of 24 